\title{
Evaluation of Pharmacologic Interventions for Weight Management in a Veteran Population
}

\author{
Sarah R. Hood, PharmD; Ashley W. Berkeley, PharmD, BCPS; and Emily A. Moore, PharmD, BCACP, NCMP
}

Background: Veterans are disproportionately impacted by weight-related morbidity: $40 \%$ of veterans are categorized as obese and an additional $38.5 \%$ are overweight. Medications are recommended as an adjunct to lifestyle and dietary changes. Guidelines recommend 7 weight management medications, including orlistat, liraglutide, phentermine, phentermine/topiramate, lorcaserin, and naltrexone/bupropion.

Methods: A single-center, retrospective chart review was conducted for patients who started weight management medications at Veteran Health Indiana in Indianapolis. The primary outcomes included total weight loss and weight loss as a percentage of baseline weight at $3,6,12$, and $>12$ months of therapy. Secondary outcomes included weight loss of $5 \%$ from baseline, rate of successful weight maintenance after initial weight loss of $5 \%$ from baseline, adverse drug reaction monitoring, and use of weight management medications across clinics at this site.
Results: The absolute weight difference over 12 months of weight management medication therapy was $15.8 \mathrm{~kg}$. At each time point, weight loss was found to be statistically significant when compared with baseline $(P<.001)$. Average weight change was greatest with orlistat $(-25.9 \mathrm{~kg})$ and naltrexone/ bupropion was associated with a gain of $2.1 \mathrm{~kg}$ over the duration of the study. A majority of the patients analyzed lost the guideline-recommended 5 to $10 \%$ from baseline while taking weight management medication.

Conclusions: Weight management medications in a veteran population produced initial weight loss consistent with previous studies. However, there is room for improvement in follow-up strategies to promote greater weight maintenance after initial weight loss. Considering the high health care costs, personal burden, and potential long-term complications associated with obesity, efforts to promote continued development of programs that support weight management and maintenance are imperative.
Sarah Hood was a PGY-1 Pharmacy Practice Resident at the time of the study; Ashley Berkeley and Emily Moore are Clinical Pharmacy

Specialists, all at Veteran Health Indiana in Indianapolis.

Correspondence:

Sarah Hood

(shood@alumni.purdue.edu)

Fed Pract. 2021;38(4). Published online May 14 doi:10.12788/fp.0117 l n the United States nearly $40 \%$ of the general population is considered to be overweight or obese, a $10 \%$ increase since the early 2000s. ${ }^{1}$ Veterans are disproportionately affected by this obesity epidemic. Forty percent of veterans are categorized as obese and an additional $38.5 \%$ are overweight. ${ }^{2}$ Patients in these weight categories experience challenges with activities of daily living and increased health risks, including heart disease, stroke, type 2 diabetes (T2DM), and certain cancers. ${ }^{1}$

The American Heart Association, the American College of Cardiology, and the Obesity Society define overweight as a body mass index (BMI) of 25 to 29.9 and obesity as a BMI $\geq 30$. Morbid obesity is defined as a BMI $\geq 35$ or $40 .^{2,3}$ Based on these BMI cutoffs, the Endocrine Society recommends diet and lifestyle as the foundation of weight management and pharmacotherapy for those with a BMI $\geq 30$ without comorbidities. In patients with a BMI $\geq 27$, weight management medications may be considered if a patient has comorbid hypertension, T2DM, dyslipidemia, metabolic syndrome, obstructive sleep apnea, or nonalcoholic fatty liver disease. Patients with BMI $>40$ are eligible for weight loss surgery. ${ }^{4}$
Lifestyle and dietary interventions are the foundation of current weight management guidelines from the Endocrine Society. ${ }^{4} \mathrm{At}$ a minimum, guidelines recommended enrolling motivated patients in a high-intensity lifestyle intervention class of at least 14 sessions in the first 6 months to reach a goal weight loss of 5 to $10 \%$ from baseline and to maintain a reduction of 3 to $5 \%$ from baseline. ${ }^{3}$ Medications are recommended as an adjunct to lifestyle and dietary changes. Most weight management medications work in the brain to stimulate satiety signaling, which helps motivated patients adhere to their dietary interventions, assist those who have been unsuccessful in earlier weight loss attempts, and help maintain weight. ${ }^{3,4}$

Guidelines recommend 7 weight management medications, including orlistat (both prescription strength and over-thecounter), liraglutide, phentermine, phentermine/topiramate, lorcaserin, and naltrexone/bupropion. Using medications to assist with weight loss increases likelihood that patients will achieve 5 to $10 \%$ weight loss from baseline. ${ }^{5,6}$ Studies looking at long-term effects of these medications on weight loss have found improvements in blood pressure (BP), biomarkers for 
cardiovascular disease, and T2DM-related comorbidities. ${ }^{3,5,7}$

Positive effects on comorbidities have been found to be related to drug class and mechanism of action (MOA); those that also are approved for T2DM have demonstrated the most favorable cardiovascular effects. ${ }^{7}$ Other medications that work as stimulants or as modulators of serotonin pathways are associated with increased risks, prompting the US Food and Drug Administration (FDA) to remove some medications from the market. ${ }^{78}$ In January 2020, lorcaserin was taken off the market because of increased risk of cancer found in postmarketing surveillance. ${ }^{9}$ The benefit of weight loss must be weighed against the risk of medication use.

Monthly follow-up is recommended with weight management medications in the beginning to assess safety and efficacy; medications should be discontinued if weight loss is inadequate in the first 3 months. ${ }^{1,3,4} \mathrm{Lim}$ ited studies have assessed the long-term use of weight management medications in a realworld setting. Medications are prescribed for weight management at Veteran Health Indiana (VHI) in outpatient clinics, including primary care, endocrinology, and gastrointestinal (GI) specialties. However, prescribing practices, outcomes, and adherence to guideline recommendations have not been studied. Data from this study will be used to better understand how VHI can serve its veterans through diet, lifestyle, and pharmacologic interventions.

\section{METHODS}

We conducted a single-center, retrospective chart review for patients started on weight management medications at VHI. A patient list was generated based on prescription fills from June 1, 2017 to June 30, 2019. All data were obtained using the Computerized Patient Record System and patients were not contacted. This study was approved by the Indiana University Health Institutional Review Board and the VHI Research and Development Committee.

At the time of study, orlistat, liraglutide, phentermine/topiramate, lorcaserin, and naltrexone/bupropion were available at $\mathrm{VHI}$ for patients who met the criteria. All patients must have been enrolled in dietary and lifestyle management to be approved
TABLE 1 Baseline Characteristics $(\mathrm{N}=96)^{\mathrm{a}}$

\begin{tabular}{|c|c|}
\hline Characteristics & Results \\
\hline Male, No. (\%) & $80(83.3)$ \\
\hline Age, mean (SD), y & $53(12.8)$ \\
\hline \multicolumn{2}{|l|}{ Race, No. (\%) } \\
\hline White & $83(86.5)$ \\
\hline African American & $9(9.4)$ \\
\hline American Indian/Alaska Native & $2(2.1)$ \\
\hline Other & $2(2.1)$ \\
\hline Body mass index, mean (SD) & $41.1(7.4)$ \\
\hline Weight, mean (SD), kg & $130.0(24.7)$ \\
\hline Glycated hemoglobin level, mean (SD) & $6.2(1.2)$ \\
\hline \multicolumn{2}{|l|}{ Blood pressure, mean (SD), $\mathrm{mm} \mathrm{Hg}$} \\
\hline Systolic & $131.7(14.9)$ \\
\hline Diastolic & $79.1(12.0)$ \\
\hline \multicolumn{2}{|l|}{ Cholesterol, mean (SD), mg/dL } \\
\hline Total & $158.7(33.4)$ \\
\hline Low-density lipoprotein & $87.2(31.3)$ \\
\hline High-density lipoprotein & $39.1(8.8)$ \\
\hline Triglycerides & $168.9(8.8)$ \\
\hline Thyroid-stimulating hormone, mean (SD), mU/L & $2.2(1.4)$ \\
\hline Heart rate, mean (SD), beats per min & $79.2(14.0)$ \\
\hline \multicolumn{2}{|l|}{ Comorbidities, No. (\%) } \\
\hline Diabetes mellitus & $38(39.6)$ \\
\hline Hypertension & $63(65.6)$ \\
\hline Hyperlipidemia & $61(63.5$ \\
\hline Cardiovascular disease & $11(11.5)$ \\
\hline Arrhythmias & $6(6.3)$ \\
\hline Hypothyroidism & $12(12.5)$ \\
\hline Hyperthyroidism & $1(1.0)$ \\
\hline Psychiatric diagnosis & $48(50)$ \\
\hline Substance use disorder (nonopioid) & $22(22.9)$ \\
\hline Cholelithiasis & $1(1.0)$ \\
\hline Glaucoma & $3(3.1)$ \\
\hline Opioid use disorder & $1(1.0)$ \\
\hline Cancer & $4(4.2)$ \\
\hline Fatty liver disease & $2(2.1)$ \\
\hline
\end{tabular}

for these medications. The US Department of Veterans Affairs (VA) MOVE! weight management program, designed by the VA National Center for Health Promotion and Disease Promotion, is available at VA sites throughout the country. After MOVE! orientation, patients can participate in group or individual 12 -week programs that 
TABLE 2 Medications for Comorbid Conditions at Baseline $(\mathrm{N}=96)$

\begin{tabular}{lc} 
Medications & Patients, No. (\%) \\
\hline Insulin & $20(20.1)$ \\
\hline Sulfonylurea & $5(5.2)$ \\
\hline Metformin & $25(26.0)$ \\
\hline SGLT2 inhibitor & $5(5.2)$ \\
\hline GLP-1 agonist & $1(1.0)$ \\
\hline Diuretic & $27(28.1)$ \\
\hline$\beta$ blocker & $24(25.0)$ \\
\hline Anticonvulsant & $22(22.9)$ \\
\hline Antipsychotic & $4(4.2)$ \\
\hline Tricyclic antidepressant & $2(2.1)$ \\
\hline Hormone replacement & $2(2.1)$
\end{tabular}

include weigh ins, goal-setting strategies, meal planning, and habit modification support. If patients are unable to meet in person, phone and other telehealth opportunities are available.

Patients were included in the study if they received a prescription of any 1 of the 5 available medications during the enrollment period. Patients were excluded if they received a prescription from or were treated by a civilian health care provider, if they never used the medication, or if their weight loss was attributed to a cancer diagnosis. These criteria produced 86 patients of whom 96 unique weight loss prescriptions were generated. Data were collected for each instance of medication use so that some patients were included multiple times. In this case, data collection for the failed medication ended when failure was documented, and new data points began when new medication was prescribed; all data collected were per medication, not per patient. This method was used to account for medication failure and provide accurate weight loss results based on medication choice within this institution.

The primary outcomes included total weight loss and weight loss as a percentage of baseline weight at 3, 6, 12, and
$>12$ months of therapy. Secondary outcomes included weight loss of 5\% from baseline, rate of successful weight maintenance after initial weight loss of $5 \%$ from baseline, adverse drug reaction (ADR) monitoring, and use of weight management medications across clinics at VHI.

Demographic data included race, age, sex, baseline weight, BMI, and comorbid medical conditions. Comorbidities were collected based on the most recent primary care clinical note before initiating medication. Medication data collected included medications used to manage comorbidities. Data related to weight management medication included prescribing clinic, reason for medication discontinuation, or bariatric surgery intervention if applicable.

Efficacy outcome data included weight and BMI across therapy duration. Safety outcomes data included heart rate, $\mathrm{BP}$, and ADRs that resulted in medication discontinuation as documented in the electronic health record (EHR).

We used descriptive statistics, including mean, standard deviation (SD), range, and percentage. For continuous data, KruskalWallis tests were used because of nonparametric data distribution among the different medications with a prespecified $\alpha=0.05$. With the observed sample sizes and SDs in this study, post hoc poststudy power calculations showed that the study had $80 \%$ power at a 5\% significance level to detect weight changes of $8.6 \mathrm{~kg}, 7.3 \mathrm{~kg}$, and $12.4 \mathrm{~kg}$ at 3,6 , and 12 months, respectively, using nonparametric tests.

\section{RESULTS}

A total of 86 patients were identified based on prescription fills, which produced 99 unique instances of medication use. Of the 99 identified, 3 met exclusion criteria and were not included in the final analysis. Among included veterans, 16 were female and 80 were male (Table 1). Most of those included identified as White race (86\%), male $(83 \%)$, and mean age 53 years. At baseline, mean weight was $130 \mathrm{~kg}$ and mean BMI 41.

\section{Comorbidities and Medication Use}

Hypertension (66\%), hyperlipidemia (64\%), and psychiatric diagnoses (50\%) were most common comorbid conditions. Substance use 
(23\%) and T2DM (40\%) were the most common comorbidities influencing medication choice. Substance use evaluation included amphetamines and cocaine for this analysis.

Phentermine/topiramate is the preferred first-line agent unless patients have contraindications for use, in which case naltrexone/bupropion is recommended, based on guidelines for weight management medications within the VHI system. However, for patients with comorbid T2DM, liraglutide is preferred because of its beneficial effects for both weight loss and blood glucose control. ${ }^{2}$ Most patients at VHI were started on liraglutide $(44 \%)$ or phentermine/topiramate (42\%), which was in line with recommendations. Our sample included $\geq 1$ prescription for each medication available at our facility, although the number of patients on each medication was not equal. Of note, the one patient taking lorcaserin at the time of study discontinued therapy in response to recent FDA guidance. ${ }^{9}$

Medications for comorbid conditions could contribute to weight gain. Of the patient sample, $\beta$ blockers $(n=24)$ and anticonvulsants, including gabapentin and pregabalin $(\mathrm{n}=22)$ were the most common Other medications that could have contributed to weight gain included sulfonylureas $(n=5)$, antipsychotics $(n=4)$, tricyclic antidepressants $(n=2)$, and hormone replacement therapies $(\mathrm{n}=2)$.

\section{Primary Outcomes}

The mean weight of participants dropped from 129.9 to $114.2 \mathrm{~kg}$ over the 12 months of weight management medication therapy for a absolute difference of $15.8 \mathrm{~kg}$ (Figure 1 and eTable 1 available at doi:10.12788 /fp.0117). Weight loss was recorded at 3, 6, 12 , and $>12$ months of weight management therapy. At each time point, weight loss was statistically significant $(P<.001)$ compared with baseline (Table 2), even though not every patient had weight loss records at each time point.

When classified by medication choice, mean change in weight was orlistat $-25.9 \mathrm{~kg}(\mathrm{n}=5)$; lorcaserin $-22.5 \mathrm{~kg}$ $(\mathrm{n}=1)$; liraglutide $-10.3 \mathrm{~kg}(\mathrm{n}=43)$; phentermine/topiramate $-5.0 \mathrm{~kg}(\mathrm{n}=42)$; and naltrexone/ bupropion $+2.1 \mathrm{~kg}(\mathrm{n}=5)$ over the duration of the study (eTable 2 available at
FIGURE 1 Mean Weight Over Time

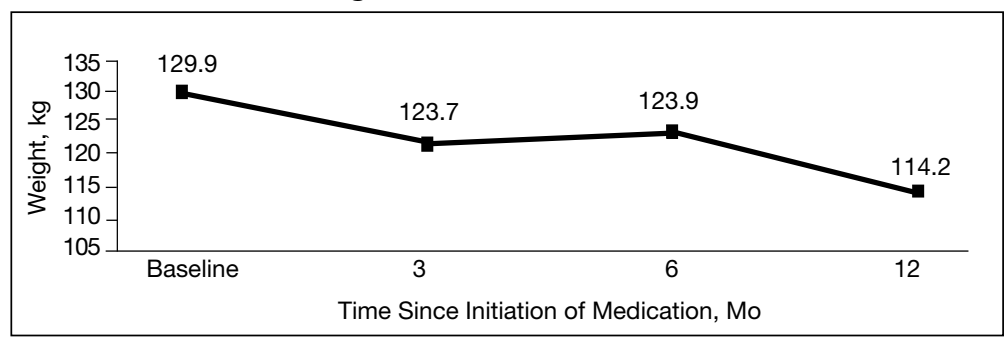

doi:10.12788/fp.0117). Patients receiving orlistat and liraglutide had increasing weight loss across the duration of treatment compared with those on lorcaserin who had initial weight gain before weight loss. Phentermine/topiramate showed initial weight loss that tapered off, and naltrexone/bupropion demonstrated initial weight loss with eventual weight gain at study end point.

\section{Secondary Outcomes}

More than one-half of the patients analyzed lost 5 to $10 \%$ from baseline while taking weight management medication. Fifty-six (59\%) patients lost 5\% at any point while on therapy (Table 3). Among those patients, $31(32 \%)$ lost $\geq 10 \%$ from baseline. When looking at success related to guideline recommendations, 32 (38\%) patients lost 5\% from baseline in the first 3 months of medication therapy.

Among patients who lost at least 5\% from baseline, we performed further analysis to assess weight maintenance of 3 to $5 \%$ from baseline for 12 months. In the 12 months after initial weight loss, the number of patients who were able to maintain their weight loss steadily declined (Figure 2 ). In the end, 17 (30\%) of patients who achieved 5 to $10 \%$ weight loss at baseline were able to maintain weight loss. Only $18 \%$ of study patients were able to achieve guideline-directed weight loss and maintain that weight for 12 months.

We found that most of our prescriptions $(n=50)$ were entered by the endocrinology department in conjunction with the MOVE! program (eTable 3 available at doi:10.12788/fp.0117). All 4 of our primary care clinics prescribed weight loss medication; however, 1 clinic prescribed the most. Other prescriptions came from communitybased outpatient clinics or other specialties, 
TABLE 3 Patients Who Achieved $\geq 5 \%$ Weight Loss From Baseline $(\mathrm{N}=96)$

\begin{tabular}{ll} 
Weight Loss, \% & No. (\%) \\
\hline $5 \%$ weight loss at 3 mo & $32(37.5)$ \\
\hline $5 \%$ weight loss ever & $56(58.3)$ \\
\hline $10 \%$ weight loss ever & $31(32.3)$
\end{tabular}

aThose who lost $10 \%$ weight loss ever are also included in the $5 \%$ weight loss ever measurement.

including gastroenterology, orthopedics, and sleep medicine.

Nineteen (18\%) patients experienced an adverse event $(\mathrm{AE})$ that led to medication discontinuation, which was recorded in their chart (eTable 4 available at doi:10.12788/fp.0117). Most common AEs were GI upset with liraglutide or orlistat or dull aching and pain with phentermine/ topiramate. Two severe AEs occurred: One patient experienced a change in mental health status and suicide attempt with naltrexone/bupropion; and 1 patient discontinued phentermine/topiramate because of a change in neurologic status.

Primarily medications were stopped because of inadequate weight loss $(n=13)$, and most patients tried additional medications. However, 1 medication failure resulted in sleeve gastrectomy. Other reasons for medication discontinuation included missed MOVE! appointments, patient lost to followup, and patient-elected discontinuation.

\section{DISCUSSION}

This study evaluated the use and outcomes of weight management medication among veterans at VHI. The study aimed to better understand the efficacy and safety of these medications while exposing potential weaknesses in care and to promote avenues to improve weight loss and maintenance.

Clinical trials for weight management medications reported weight loss of 8 to $10 \mathrm{~kg}$ over 56 weeks: 21 to $63 \%$ of patients losing at least $5 \%$ from baseline weight. ${ }^{10-14}$ The findings from our study found a higher average weight loss $(-15.8 \mathrm{~kg})$ than that reported in trials and a consistent percentage of patients (58.3\%) who achieved at least $5 \%$ weight loss. It is promising to see that when used in a noncontrolled set- ting, these medications were able to produce weight loss consistent with results seen in large, controlled trials.

Pi-Sunyer and colleagues found continued weight loss after the initial 5\% weight loss to an eventual $10 \%$ weight loss in many patients. ${ }^{10}$ Additionally, Smith and colleagues found that nearly $68 \%$ of their participants who took lorcaserin were able to maintain 3 to $5 \%$ weight loss over 12 months. ${ }^{13}$ Sjöström and colleagues acknowledged that many patients taking orlistat for an extended period began to gain weight, although at one-half the rate than that seen in the placebo group. ${ }^{12}$ This study found that fewer patients were able to maintain their weight loss over 12 months, with only $30 \%$ of patients maintaining 3 to $5 \%$ weight loss from baseline. This difference in weight maintenance likely was because of the uncontrolled nature of this study. Once patients reach their initial weight loss goal, even the most motivated patients will have trouble maintaining that weight. ${ }^{4}$ Despite the challenges associated with maintaining weight loss, the quality of life benefits patients gained and potential reductions in health care spending support using resources to improve these outcomes. ${ }^{2,14,15}$

Pi-Sunyer and colleagues reported high incidences of nausea (40\%), vomiting $(16 \%)$, diarrhea $(21 \%)$, and constipation (20\%) with liraglutide. ${ }^{10}$ Sjöström and colleagues reported $7 \%$ of patients experienced GI upset with orlistat. ${ }^{12}$ Comparatively, only $17 \%$ of our patients reported AEs that required discontinuation, including GI upset. One patient in our study discontinued naltrexone/bupropion because of a significant change in mental status and suicide attempt. Clinical trials did not report a greater risk of depression or suicidality compared with placebo; however, there is a warning on the labeling of naltrexone/bupropion for increased suicidality with the use of antidepressant agents. ${ }^{16,17}$ The neurologic AE that required discontinuation of phentermine/ topiramate at our institution is unique based on published information. ${ }^{11,18}$

The data from this study reinforced the observation that weight maintenance is the most challenging aspect of weight loss. Although our data showed clinically meaningful weight loss from baseline, many patients 
regained their weight, and some exceeded their baseline weight. Beyond providing these medications, this evidence suggests the need for close, continued follow-up through patients' weight loss journey.

\section{Limitations}

Because this is a retrospective chart review, data collection was influenced by and limited to information that had been recorded in the EHR. AEs that resulted in medication discontinuation were assessed from the patient's chart, which might not be correct if providers did not update the records. Follow-up was not always scheduled at regular intervals after medication initiation, resulting in varying sample numbers at each time point, potentially interfering with true weight loss averages. Although not included in this analysis, it might be beneficial to evaluate adherence to recommendations for follow-up with laboratory and weight monitoring to better capture where future monitoring can be improved. Second, there was an unbalanced number of patients taking each medication. Specifically, we saw a change in weight with orlistat that exceeded what is consistently seen in larger, more controlled trials. Although this is an effect of the real world, small sample sizes cannot be generalized to the larger population and might result in data reflecting that of an outlier. Last, there is a lack of generalizability because of the veteran population demographic, which is more male and lacks ethnic diversity. This study also was carried out at a single, educational tertiary medical center, which might not apply to all populations.

\section{CONCLUSIONS}

Despite the limitations discussed, this study shows that the use of weight management medications in a general veteran population produces initial weight loss consistent with previous studies. However, there is room for continued improvement in follow-up strategies to promote greater weight maintenance after initial weight loss. Considering the high health care costs, personal burden, and potential long-term complications associated with obesity, efforts to promote development of programs that support weight management and maintenance are imperative.
FIGURE 2 Participants Who Maintained 3 to 5\% Weight Loss Over Time

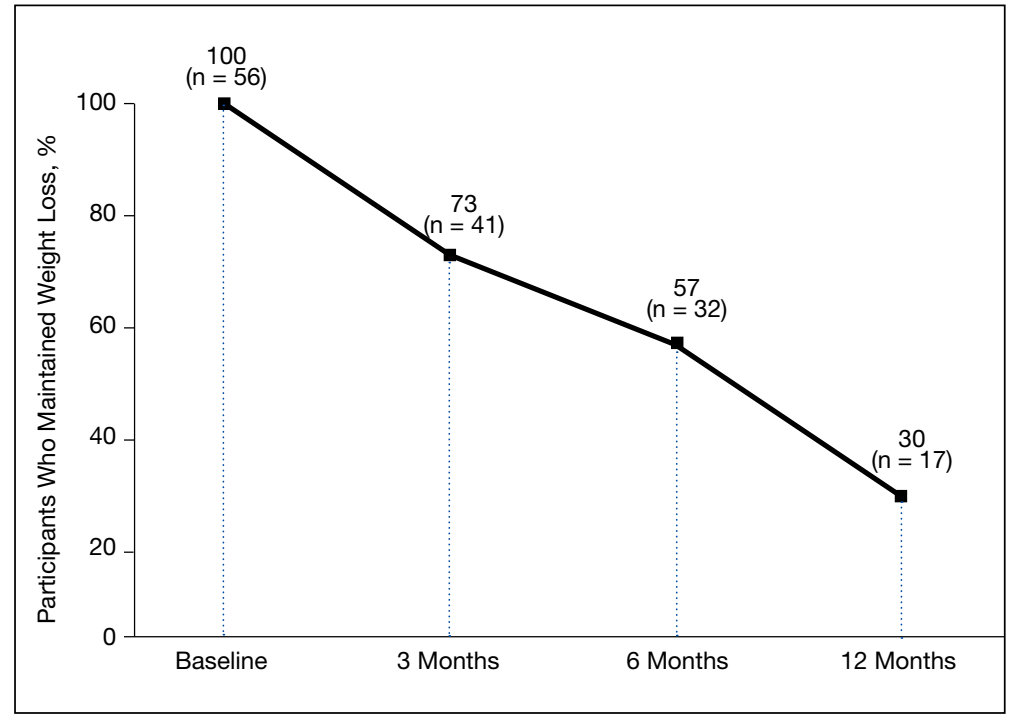

Acknowledgment

This material is the result of work supported with resources and the use of facilities at Veteran Health Indiana.

\section{Author disclosures}

The authors report no actual or potential conflicts of interest with regard to this article.

\section{Disclaimer}

The opinions expressed herein are those of the authors and do not necessarily reflect those of Federal Practitioner, Frontline Medical Communications Inc., the US Government, or any of its agencies. This article may discuss unlabeled or investigational use of certain drugs. Please review the complete prescribing information for specific drugs or drug combinations-including administering pharmacologic therapy to patients.

\section{References}

1. Centers for Disease Control and Prevention. Adult obesity facts. Accessed April 2020. https://www.cdc.gov/obesity /data/adult.html

2. The Management of Overweight and Obesity Working Group. VA/DoD Clinical Practice Guideline for Screening and Management of Overweight and Obesity. Accessed March 13, 2021. https://www.healthquality.va.gov/guide lines/CD/obesity/VADoDCPGManagementOfOverweight AndObesityFinal.pdf

3. Jensen MD, Ryan DH, Apovian CM, et al; American College of Cardiology/American Heart Association Task Force on Practice Guidelines; Obesity Society. 2013 AHAVACC/TOS guideline for the management of overweight and obesity in adults: a report of the American College of Cardiology/ American Heart Association Task Force on Practice Guidelines and the Obesity Society. J Am Coll Cardiol. 2014;63(25, pt B):2985-3023. doi:10.1016/j.jacc.2013.11.004

4. Apovian CM, Aronne LJ, Bessesen DH, et al; Endocrine Society. Pharmacological management of obesity: an Endocrine Society clinical practice guideline. J Clin Endocrinol Metab 2015;100(2):342-362. doi:10.1210/jc.2014-3415

5. Rucker D, Padwal R, Li SK, Curioni C, Lau DCW. Long term pharmacotherapy for obesity and overweight: updated meta-analysis. BMJ. 2007;335(7631):1194-1199. doi:10.1136/bmj.39385.413113.25

6. Siebenhofer A, Winterholer, S, Jeitler K, et al. Long-term 
effects of weight-reducing drugs in people with hypertension. Cochrane Database Syst Rev 2021;1:CD007654. doi:10.1002/14651858.CD007654.pub5

7. Bramante CT, Raatz S, Bomber EM, Oberle MM, Ryder JR. Cardiovascular risks and benefits of medications used for weight loss. Front Endocrinol (Lausanne). 2020;10:883. doi:10.3389/fendo.2019.00883

8. Christensen R, Kristensen PK, Bartels EM, Bliddal $H$, Astrup A. Efficacy and safety of the weight-loss drug rimonabant: a meta-analysis of randomized trials. Lancet. 2007;370(9600):1706-1713. doi:10.1016/S0140-6736(07)61721-8

9. US Food and Drug Administration. FDA requests the withdrawal of the weight-loss drug Blevique, Belvique XR (lorcaserin) from the market. Accessed April 2020. https:// www.fda.gov/drugs/drug-safety-and-availability/fda -requests-withdrawal-weight-loss-drug-belviq-belviq -xr-lorcaserin-market

10. Pi-Sunyer X, Astrup A, Fujioka K, et al; SCALE Obesity and Prediabetes NN8022-1839 Study Group. A randomized, controlled trial of $3.0 \mathrm{mg}$ of liraglutide in weight management. N Engl J Med. 2015;373(1):11-22. doi:10.1056/NEJMoa1411892

11. Gadde KM, Allison DB, Ryan DH, et al. Effects of low-dose, controlled-release phentermine plus topiramate combination on weight and associated comorbidities in overweight and obese adults (CONQUER): a randomized, placebo-controlled, phase 3 trial. Lancet. 2011;377(9774):1341-1352.
doi:10.1016/S0140-6736(11)60205-5

12. Sjöström L, Rissanen A, Andersen T, et al. Randomised placebo-controlled trial of orlistat for weight loss and prevention of weight regain in obese patients. European Multicentre Orlistat Study Group. Lancet. 1998;352(9123):167-172. doi:10.1016/s0140-6736(97)11509-4

13. Smith SR, Weissman NJ, Anderson CM, et al; Behaviora Modification and Lorcaserin for Overweight and Obesity Management (BLOOM) Study Group. Multicenter, placebocontrolled trial of lorcaserin for weight loss. N Engl J Med. 2010;363(3):245-256. doi:10.1056/NEJMoa0909809

14. Warkentin LM, Das D, Majumdar SR, Johnson JA, Padwal RS. The effect of weight loss on health-related quality of life: systematic review and meta-analysis of randomized trials. Obes Rev. 2014;15(3):169-182. doi:10.1111/obr.12113

15. Finkelstein EA, Trogdon JG, Cohen JW, Dietz W. Annual medical spending attributable to obesity: payerand service-specific estimates. Health Aff (Millwood). 2009;28(5):w822-831. doi:10.1377/hlthaff.28.5.w822

16. Greenway FL, Fujioka K, Plodkowski RA, et al; COR-I Study Group. Effect of naltrexone plus bupropion on weight loss in overweight and obese adults (COR-I): a multicenter, randomized, double-blind, placebo-controlled phase 3 trial. Lancet. 2010;376(9741):595-605. doi:10.1016/S0140-6736(10)60888-4

17. Contrave. Prescribing information. Nalpropion Pharmaceuticals, Inc; 2019.

18. Qsymia. Prescribing information. VIVUS Inc; 2018. 
eTABLE 1 Mean Weight Over Time

\begin{tabular}{lccc} 
Periods $^{\text {b }}$ & Patients, No. & Weight, mean (SD), kg & $\boldsymbol{P}$ value \\
\hline Baseline & 96 & $129.9(24.7)$ & \\
\hline $3 \mathrm{mo}$ & 72 & $123.7(24.8)$ & $<.001$ \\
\hline $6 \mathrm{mo}$ & 98 & $123.9(22.8)$ & $<.001$ \\
\hline $12 \mathrm{mo}$ & 36 & $114.3(19.9)$ & $<.001$ \\
\hline Current & 81 & $121.1(24.2)$ & $<.001$
\end{tabular}

aBaseline characteristics were taken at the time of first prescription for patients receiving $>1$ medication. This method was based on findings that baseline characteristics may vary given duration between medications used and other changes in comorbidities across treatment time lines.

bWeight at 3, 6, and 12 months and current compared with baseline.

eTABLE 2 Weight Loss Over Time Compared With Baseline

\begin{tabular}{|c|c|c|c|c|c|}
\hline Medications & $\begin{array}{l}\text { Patients, } \\
\text { No. (\%) }\end{array}$ & $\begin{array}{l}\text { Weight Change } \\
\text { at } 3 \mathrm{mo}(\mathrm{kg})(\mathrm{n}=80)\end{array}$ & $\begin{array}{l}\text { Weight Change } \\
\text { at } 6 \mathrm{mo}(\mathrm{kg})(\mathrm{n}=72)\end{array}$ & $\begin{array}{l}\text { Weight Change } \\
\text { at } 12 \mathrm{mo}(\mathrm{kg})(\mathrm{n}=36)\end{array}$ & $\begin{array}{c}\text { Average Current } \\
\text { Weight (kg) }(n=81)\end{array}$ \\
\hline Lorcaserin, mean (SD) & $1(1)$ & $+0.4(0.0)$ & $-5.6(0.0)$ & $-17.0(0.0)$ & $-22.5(0.0)$ \\
\hline Orlistat, mean (SD) & $5(6)$ & $-3.0(10.9)$ & $-7.9(11.9)$ & $-9.7(13.5)$ & $-25.9(21.5)$ \\
\hline Liraglutide, mean (SD) & $43(44)$ & $-2.7(24.2)$ & $-2.7(20.7)$ & $-9.4(22.1)$ & $-10.3(22.9)$ \\
\hline Naltrexone/bupropion, mean (SD) & $5(7)$ & $-2.9(5.78)$ & $-6.2(6.6)$ & $-0.5(6.7)$ & $+2.1(5.8)$ \\
\hline $\begin{array}{l}\text { Phentermine/topiramate, mean } \\
\text { (SD) }\end{array}$ & $42(42)$ & $-14.4(27.6)$ & $-11.1(26.5)$ & $-11.8(18.7)$ & $-5.0(59.1)$ \\
\hline
\end{tabular}

eTABLE 3 Prescribing Clinic ( $\mathrm{N}=96)$

\begin{tabular}{lc} 
Clinics & No. \\
\hline Primary care & 42 \\
\hline Specialty clinics & \\
Endocrinology/MOVE! & 50 \\
Gastroenterology & 2 \\
Orthopedics & 1 \\
Sleep medicine & 1
\end{tabular}

eTABLE 4 Discontinuation and Safety Event Reporting ( $\mathrm{N}=96)$

\begin{tabular}{lc} 
Reasons Discontinued & No. (\%) \\
\hline Mild adverse event & $17(17.7)$ \\
\hline Severe adverse event & $2(2.1)$ \\
\hline Missed MOVE! appointment & $9(9.4)$ \\
\hline Medication failure & $13(13.5)$ \\
\hline Patients lost to follow-up & $12(12.5)$ \\
\hline Patient-driven discontinuation & $3(3.1)$
\end{tabular}

